\title{
COMPLICACIONES CARDIOPULMONARES ASOCIADAS A PROPOFOL VERSUS SEDACIÓN TRADICIONAL PARA PROCEDIMIENTOS ENDOSCÓPICOS DIGESTIVOS EN ADULTOS: REVISIÓN SISTEMÁTICA Y METAANÁLISIS DE ESTUDIOS CLÍNICOS RANDOMIZADOS (ECR)*
}

\author{
Drs. Javiera Carmona B. ${ }^{1}$, Hernán Auad A. ${ }^{1}$, \\ Fernando Altermatt C. ${ }^{1}$, Jorge Dagnino $\mathrm{S} .{ }^{1}$ \\ ${ }^{1}$ Departamento de Anestesiología Hospital Clínico Pontificia Universidad Católica de Chile. \\ Santiago, Chile.
}

\begin{abstract}
Cardiopulmonary complications associated with traditional versus propofol sedation for gastrointestinal endoscopic procedures in adults: systematic review and metaanalysis of randomized clinical trials (RCT)
\end{abstract}

Background: For sedation in endoscopy, propofol offers advantages for its fast recovery profile compared with traditional sedation (opioids and/or benzodiazepines). However, their use may be associated with deeper levels of sedation, increasing the risk of complications. Aims: To evaluate the safety of propofol compared with traditional endoscopic procedures. To analyze influence of operator sedation in endoscopic procedures. Materials and Methods: ECR comparing cardiopulmonary complications (hypoxia, hypotension, bradycardia) between propofol and traditional sedation in adults undergoing endoscopy, were included. We searched MEDLINE, EMBASE, Cochrane Controlled Trials Registry, LILACS. Relative risk (RR) was calculated. The data were analyzed using Rev Man v.5.3 (The Cochrane Collaboration). Results: 8 ECR were selected, totaling 739 patients; of these, 381 received propofol and 358 traditional sedation. An anesthesiologist administered sedation in 3 of the 8 papers, in patients with greater comorbidity and deeper level of sedation. Compared with traditional sedation, the RR of developing hypoxia, hypotension or bradycardia with propofol was 0.78 (95\% CI, 0.56-1.08), 1.37 (95\% CI, 0.82-2.28), 0.57 (95\% CI, 0.27-1.24) respectively. The RR of hypoxia in using propofol by an anesthesiologist was 1.59 (95\% CI, 0.38-6.72) and non-anesthesiologist was 0.75 (95\% CI, 0.53-1.05). No statistically significant differences between propofol and traditional sedation for the analyzed adverse effects were found. Conclusions: Use of propofol compared with traditional sedation for endoscopic procedures do not increase the risk of cardiopulmonary complications. There were no significant differences when analyzing the professional in charge of sedation.

Key words: Sedation, endoscopy, propofol, cardiopulmonary complications.

*Recibido el 6 de marzo de 2015 y aceptado para publicación el 11 de mayo de 2015.

Los autores no refieren conflictos de interés.

Correspondencia: Dr. Fernando Altermatt C.

fernando.altermatt@gmail.com 


\section{Resumen}

Introducción: Para sedación en endoscopia, el propofol ofrece ventajas por su rápido perfil de recuperación comparado con sedación tradicional (opiodes y/o benzodiacepinas). Sin embargo, su uso podría estar asociado a niveles de sedación más profunda, aumentando el riesgo de complicaciones. Objetivos: Evaluar la seguridad del propofol comparado con sedación tradicional para procedimientos endoscópicos. Analizar la influencia del operador de la sedación en procedimientos endoscópicos. Materiales y Métodos: Se incluyeron ECR que compararan complicaciones cardiopulmonares (hipoxia, hipotensión, bradicardia) entre propofol y sedación tradicional, en adultos sometidos a endoscopia. Se buscó en: MEDLINE, EMBASE, Cochrane Controlled Trials Registry, LILACS. Se calculó riesgo relativo (RR). Los datos se analizaron con RevMan v.5.3 (The Cochrane Collaboration). Resultados: 8 ECR fueron seleccionados, sumando 739 pacientes; de ellos, 381 recibieron propofol y 358 sedación tradicional. Un anestesiólogo administró la sedación en 3 de los 8 trabajos, en pacientes con mayor comorbilidad y nivel de sedación más profunda. Comparado con sedación tradicional, el RR de desarrollar hipoxia, hipotensión o bradicardia con propofol fue de 0,78 (IC 95\%, 0,561,08), 1,37 (IC 95\%, 0,82-2,28), 0,57 (IC 95\%, 0,27-1,24) respectivamente. El RR de hipoxia al usar propofol por anestesiólogo fue de 1,59 (IC 95\%, 0,38-6,72) y por no-anestesiólogo fue de 0,75 (IC 95\%, 0,53-1,05). No se encontraron diferencias estadísticamente significativas entre propofol y sedación tradicional para los efectos adversos analizados. Conclusiones: El uso de propofol comparado con sedación tradicional para procedimientos endoscópicos no aumenta el riesgo de complicaciones cardiopulmonares. No hubo diferencias significativas al analizar el profesional a cargo de la sedación.

Palabras clave: Sedación, endoscopia, propofol, complicaciones cardiopulmonares.

\section{Introducción}

Existen diversas alternativas para dar sedación en procedimientos endoscópicos. Entre ellas, el propofol ofrece ventajas por su rápido perfil de recuperación y está siendo cada vez más usado comparado con sedación tradicional (opiodes y/o benzodiacepinas $)^{1}$. Sin embargo, su uso podría estar asociado a niveles de sedación más profunda, lo que aumentaría el riesgo de eventos adversos tales como depresión respiratoria, problemas con la permeabilidad de la vía aérea, hipotensión o bradicardia. Por este motivo, en general se recomienda que el propofol sea administrado por anestesiólogos o personal entrenado en manejo avanzado de reanimación y control de la vía aérea durante la sedación ${ }^{2}$. Este es un punto no exento de controversia; en un trabajo descriptivo Rex et al., sugieren que equipos de endoscopistas y enfermeras entrenadas en manejo de vía aérea y sedación pueden administrar de manera segura propofol para procedimientos endoscópicos ${ }^{3}$.

En el año 2011, una revisión sistemática y metaanálisis publicado en The Cochrane Collaboration $^{4}$, comparó el uso de propofol versus sedación tradicional para colonoscopias exclusivamente, concluyendo que el propofol aumenta la satisfacción de los pacientes sin aumentar la tasa de eventos adversos. Los autores además indican que son necesarios ECR de mayor calidad metodológica que comparen la influencia del operador de la sedación en colonoscopias.

No hay una revisión sistemática actualizada que compare complicaciones cardiopulmonares asocia- das a propofol versus sedación tradicional en procedimientos endoscópicos, así como tampoco hay un metaanálisis que compare la influencia del operador de la sedación.

El objetivo primario de esta revisión es evaluar la seguridad del propofol comparado con sedación tradicional para procedimientos endoscópicos digestivos en adultos, expresado como la incidencia de complicaciones cardiopulmonares (hipoxia, hipotensión, bradicardia).

El objetivo secundario es analizar la influencia del operador (anestesiólogo, personal no médico, etc.) de la sedación en procedimientos endoscópicos digestivos.

\section{Material y Método}

Los criterios de inclusión fueron los siguientes:

\section{Tipo de estudio}

ECR que comparan propofol versus sedación tradicional.

\section{Tipo de participantes}

Adultos (mayores de 18 años) sometidos a procedimientos endoscópicos digestivos.

\section{Tipo de resultados medidos}

Complicaciones cardiopulmonares: Hipotensión (presión arterial sistólica (PAS) menor a $90 \mathrm{mmHg}$ ), bradicardia (menos de 50 latidos por min), hipoxia (saturación de oxígeno menor a 90\%). 


\section{Estrategia de Búsqueda}

Se exploraron las siguientes bases de datos: MEDLINE, EMBASE, Cochrane Controlled Trials Registry, LILACS. Se buscó literatura gris en libros de resúmenes de congresos, sitios web y referencias cruzadas.

Los términos MeSH utilizados fueron "Propofol" AND "Endoscopy, Digestive System”.

\section{Selección de artículos}

Dos autores de manera independiente seleccionaron los trabajos con la misma estrategia de búsqueda. En una segunda instancia ambos investigadores escogieron los artículos según calidad metodológica (Score de $\mathrm{Jadad}^{5}$ mayor a 2). Un tercer investigador dirimió cuando hubo diferencias.

\section{Análisis estadístico}

Se calculó el riesgo relativo (RR) agrupado y se compararon los resultados de los estudios individuales usando un modelo de efecto aleatorio. Se evaluó la heterogeneidad con test de chi-cuadrado $\chi^{2}$ y el estadístico $\mathrm{I}^{2}$, (estadísticamente significativo $\mathrm{p}<0,1$ o $\mathrm{I}^{2}>50 \%$ ). Los datos se analizaron con RevMan v.5.3 (The Cochrane Collaboration).

\section{Sesgo de publicación}

Se creó un Funnel Plot con el objetivo de evaluar sesgo de publicación, para hipoxia, hipotensión y bradicardia.

\section{Resultados}

Se encontraron 506 citaciones, de las cuales 174 fueron ECR, de éstos, 30 se incluyeron para evaluar calidad metodológica, finalmente 8 artículos fueron seleccionados (Figura 1).

Un total de 739 pacientes fueron enrolados en los 8 estudios, de ellos 381 recibieron propofol y 358 sedación tradicional. Todos los pacientes correspondieron a endoscopia alta. Un anestesiólogo administró la sedación en 3 de los 8 trabajos, correspondiendo en general a pacientes con mayor comorbilidad y nivel de sedación más profunda (Tabla 1).

El método de administración de propofol en todos los trabajos incluidos fue un bolo inicial de 30 a $50 \mathrm{mg}$, seguido por bolos de 10 a $20 \mathrm{mg}$ según necesidad, determinada por el profesional a cargo de la sedación.

En cuanto a la calidad metodológica se seleccionaron artículos con Score de Jadad mayor a 2 para asegurar que los trabajos fueran aleatorizados y ciegos. La Tabla 2 resume los puntajes de cada ítem para los estudios incluidos. En general, el principal motivo de no obtener un Score de Jadad máximo fue la ausencia de adherencia a las guías CONSORT ${ }^{6}$.

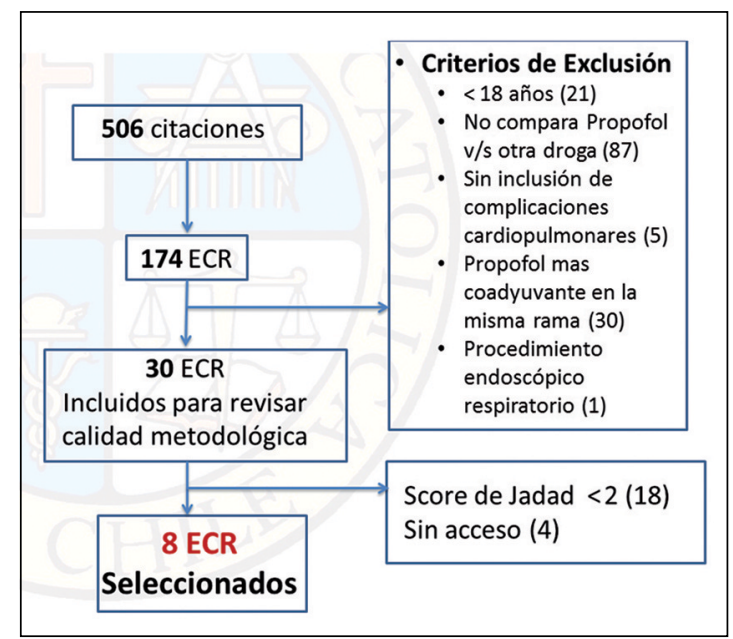

Figura 1.

Tabla 1. Características de los estudios incluidos

\begin{tabular}{|lclccl|}
\hline Estudio & n pacientes & Administrador sedación & ASA & $\begin{array}{c}\text { Procedi- } \\
\text { miento }\end{array}$ & Nivel sedación \\
\hline Agrawal $^{8}$ & 82 & Anestesiólogo & III & EDA & Mayor grupo propofol $(\mathrm{p}=0,01)$ \\
Khamaysi $^{9}$ & 61 & Anestesiólogo & III & EDA & Igual ambos grupos $(\mathrm{p}=0,1)$ \\
Kongkam $^{10}$ & 134 & Gastroenterólogo & I-III & ERCP & Desconocido \\
Riphaus $^{11}$ & 65 & Médico entrenado & III & EDA & Igual ambos grupos (sin cálculo $\mathrm{p})$ \\
Vargo $^{12}$ & 75 & Médico entrenado & I-II & ERCP/EUS & Desconocido \\
Wehrmann $^{13}$ & 198 & Médico & III-IV & ERCP & Mayor grupo propofol (sin cálculo $p)$ \\
\hline Weston $^{14}$ & 20 & Enfermera entrenada & III & EDA & Mayor grupo propofol $(\sin$ cálculo $p)$ \\
\hline Zuo $^{15}$ & 104 & Anestesiólogo & I-II & EDA & Mayor grupo propofol $(\mathrm{p}=0,01)$ \\
\hline
\end{tabular}


Tabla 2. Score de Jadad para cada estudio incluido

\begin{tabular}{|c|c|c|c|c|c|c|}
\hline Estudio & $\begin{array}{c}\text { Randomización } \\
\text { mencionada }\end{array}$ & $\begin{array}{c}\text { Randomización } \\
\text { apropiada }\end{array}$ & $\begin{array}{c}\text { Ciego } \\
\text { mencionado }\end{array}$ & $\begin{array}{c}\text { Ciego } \\
\text { apropiado }\end{array}$ & CONSORT & Puntaje \\
\hline Agrawal & 1 & 1 & 1 & 1 & 0 & 4 \\
\hline Khamaysi & 1 & 0 & 1 & 1 & 0 & 3 \\
\hline Kongkam & 1 & 0 & 1 & 1 & 0 & 3 \\
\hline Riphaus & 1 & 1 & 1 & 1 & 1 & 5 \\
\hline Vargo & 1 & 1 & 1 & 1 & 0 & 4 \\
\hline Wehrmann & 1 & 0 & 1 & 1 & 0 & 3 \\
\hline Weston & 1 & 1 & 1 & 1 & 0 & 4 \\
\hline Zuo & 1 & 1 & 1 & 1 & 1 & 5 \\
\hline
\end{tabular}

\begin{tabular}{|c|c|c|c|c|c|c|c|c|c|}
\hline Study or Subgroup & $\begin{array}{l}\text { Popo } \\
\text { Events }\end{array}$ & $\begin{array}{l}\text { fol } \\
\text { Total }\end{array}$ & \multicolumn{2}{|c|}{ Sedacion Tradicional } & Weight & \multirow{2}{*}{$\begin{array}{c}\text { Risk Ratio } \\
\text { M.H, Random, } 95 \% \mathrm{Cl} \\
\end{array}$} & \multicolumn{2}{|c|}{$\begin{array}{c}\text { Risk Ratio } \\
\text { M.H, Random, } 95 \% \mathrm{Cl}\end{array}$} & \\
\hline \multicolumn{9}{|c|}{ 1.2.1 Con anestesiólogo } & \\
\hline Agrawal, 2012 & 3 & 40 & 1 & 42 & $2.2 \%$ & $3.15[0.34,29.04]$ & - & & \\
\hline Khamaysi, 2011 & 2 & 31 & 2 & 30 & $3.0 \%$ & $0.97[0.15,6.44]$ & & & \\
\hline Zuo, 2012 & 0 & 52 & 0 & 52 & & Not estimable & & & \\
\hline Subtotal $(95 \% \mathrm{Cl})$ & & 71 & & 72 & $5.2 \%$ & $1.59[0.38,6.72]$ & & & \\
\hline Total events & 5 & & 3 & & & & & & \\
\hline \multicolumn{10}{|c|}{$\begin{array}{l}\text { Heterogeneity. Tau }{ }^{2}=0.00 ; C h i^{2}=0.63, d f=1(P=0.43) ; 1^{2}=0 \% \\
\text { Test for overall effect: } Z=0.63(P=0.53)\end{array}$} \\
\hline \multicolumn{10}{|c|}{ 1.2.2 Sin anestesiólogo } \\
\hline Kongkam, 2008 & 15 & 67 & 21 & 67 & $33.1 \%$ & $0.71[0.40,1.26]$ & $\rightarrow$ & & \\
\hline RIPHAUS, 2009 & 3 & 43 & 2 & 22 & $3.7 \%$ & $0.77[0.14,4.26]$ & & & \\
\hline Vargo, 2002 & 14 & 38 & 21 & 37 & $42.6 \%$ & $0.65[0.39,1.07]$ & $\rightarrow$ & & \\
\hline Wehrmann, 1999 & 11 & 100 & 8 & 98 & $14.3 \%$ & $1.35[0.57,3.21]$ & - & - & \\
\hline $\begin{array}{l}\text { Weston, } 2003 \\
\text { Subtotal (95\% Cl) }\end{array}$ & 0 & $\begin{array}{r}10 \\
258\end{array}$ & 1 & $\begin{array}{r}10 \\
234\end{array}$ & $\begin{array}{r}1.1 \% \\
94.8 \%\end{array}$ & $\begin{array}{r}0.33[0.02,7.32] \\
0.75[0.53,1.05]\end{array}$ & 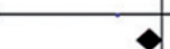 & & \\
\hline $\begin{array}{l}\text { Subtotal }(95 \% \mathrm{Cl}) \\
\text { Total events }\end{array}$ & 43 & 258 & 53 & 234 & $94.8 \%$ & $0.75[0.53,1.05]$ & & & \\
\hline \multicolumn{10}{|c|}{$\begin{array}{l}\text { Heterogeneity. Tau }{ }^{2}=0.00 ; C h \mathrm{I}^{2}=2.41, \mathrm{df}=4(P=0.66) ; \mathrm{I}^{2}=0 \% \\
\text { Test for overall effect: } Z=1.69(P=0.09)\end{array}$} \\
\hline Total $(95 \% \mathrm{Cl})$ & & 329 & & 306 & $100.0 \%$ & $0.78[0.56,1.08]$ & $\Delta$ & & \\
\hline \multirow{2}{*}{\multicolumn{6}{|c|}{$\begin{array}{l}\text { Heterogeneity. Tau }{ }^{2}=0.00 ; C h i^{2}=4.10, d f=6(P=0.66) ; I^{2}=0 \% \\
\text { Test for overall effect. } Z=1.50(P=0.13) \\
\text { Test for subaroup differences: } C h i^{2}=1.00, d f=1(P=0.32) . I^{2}=0 \%\end{array}$}} & & & & \\
\hline & & & & & & 0.005 & Favor Propofol $^{0.1}$ & Favor ST & 200 \\
\hline \multicolumn{10}{|l|}{ Caption } \\
\hline Forest plot of comp & arison: 1 & Propc & ofol v/s sed & $\mathrm{n}$ tradic & cional, o & outcome: 1.2 Desaturacion. & & & \\
\hline
\end{tabular}

Figura 2.

Comparado con sedación tradicional, el RR de desarrollar hipoxia, hipotensión o bradicardia con propofol fue de 0,78 (IC 95\%, 0,56-1.08, $\mathrm{I}^{2}=0 \%$ ), 1,37 (IC 95\%, 0,82-2,28, $\mathrm{I}^{2}=0 \%$ ), 0,57 (IC 95\%, $0,27-1,24, I^{2}=38 \%$ ) respectivamente (Figuras 2, 3 y 4). La diferencia no fue estadísticamente significativa para ninguna de las tres complicaciones evaluadas.

En el análisis de subgrupo, el RR de hipoxia al usar propofol por anestesiólogo fue de 1,59 (IC $95 \%, 0,38-6,72, \mathrm{I}^{2}=0 \%$ ) y por no-anestesiólogo fue de 0,75 (IC 95\%, 0,53-1,05, $\mathrm{I}^{2}=0 \%$ ) (Figura 2). El riesgo de hipotensión y bradicardia mantuvo igual tendencia, independiente del operador (Figuras $3 \mathrm{y}$ 4). No se encontraron diferencias estadísticamente significativas entre propofol y sedación tradicional, al analizar el profesional a cargo de sedar a los pacientes.

Se hizo un análisis de sensibilidad excluyendo uno por uno los trabajos incluidos para cada efecto adverso analizado y los resultados mantuvieron su tendencia. La Tabla 3 resume el efecto del análisis de sensibilidad al excluir uno a uno los trabajos en relación a la hipoxia. Los funnel plot no mostraron sesgo de selección para ningún resultado analizado. La Figura 5 muestra el funnel plot para hipoxia. 


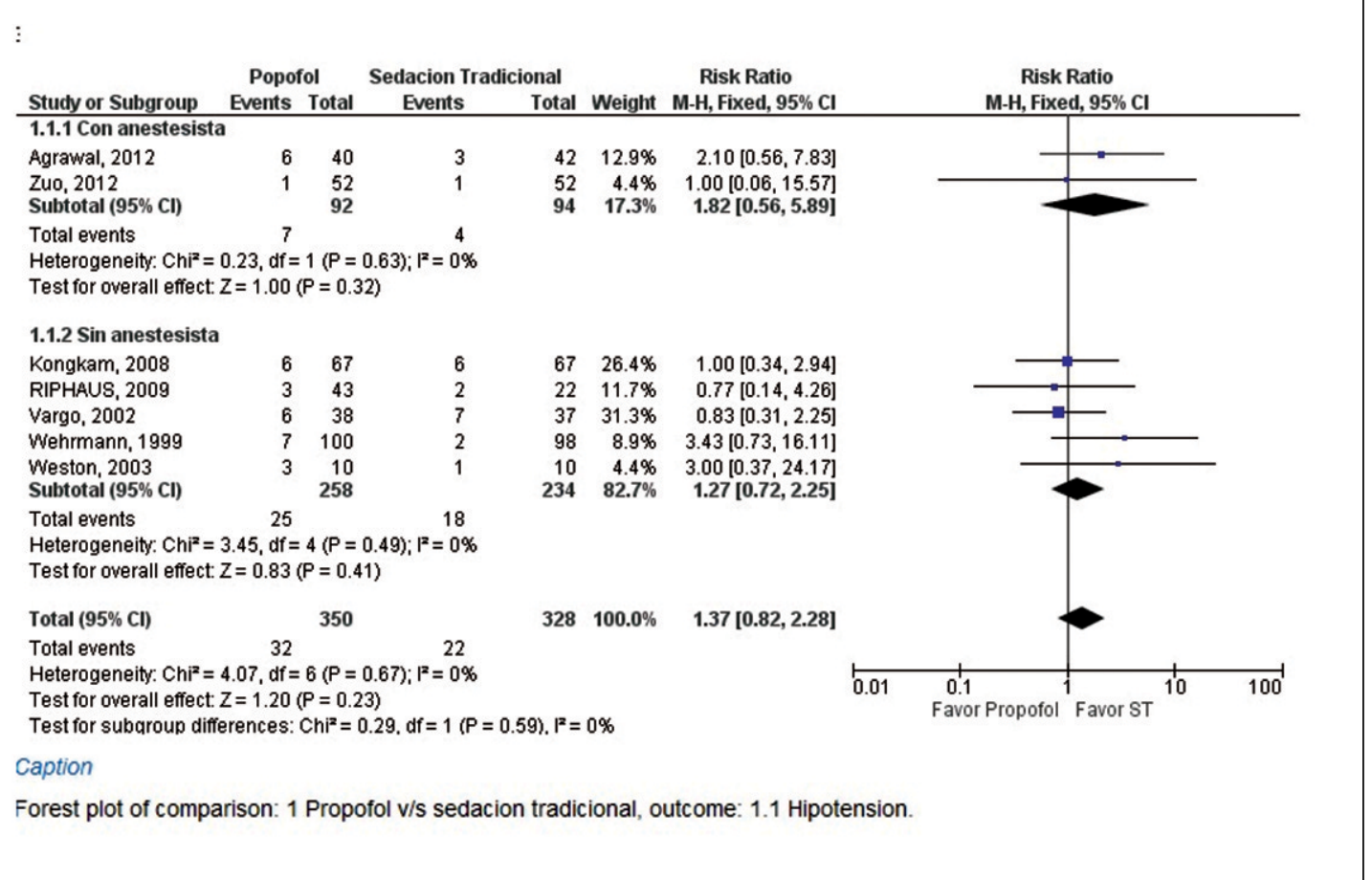

Figura 3.

\begin{tabular}{|c|c|c|c|c|c|c|c|c|c|c|}
\hline Study or Subgroup & $\begin{array}{l}\text { Popof } \\
\text { Events }\end{array}$ & fol & $\begin{array}{l}\text { Sedacion Tradi } \\
\text { Events }\end{array}$ & $\begin{array}{c}\text { ional } \\
\text { Total } \\
\end{array}$ & \multirow[t]{2}{*}{ Weight } & $\begin{array}{l}\text { Risk Ratio } \\
\text { M-H, Fixed, } 95 \% \mathrm{Cl} \\
\end{array}$ & \multicolumn{4}{|c|}{$\begin{array}{c}\text { Risk Ratio } \\
\text { M-H, Fixed, } 95 \% \mathrm{Cl}\end{array}$} \\
\hline \multicolumn{10}{|l|}{ 1.3.1 Con anestesista } & \\
\hline $\begin{array}{l}\text { Zuo, } 2012 \\
\text { Subtotal (95\% Cl) }\end{array}$ & 0 & $\begin{array}{l}52 \\
52\end{array}$ & 0 & $\begin{array}{l}52 \\
52\end{array}$ & & $\begin{array}{l}\text { Not estimable } \\
\text { Not estimable }\end{array}$ & & & & \\
\hline $\begin{array}{l}\text { Total events } \\
\text { Heterogeneity. Not a } \\
\text { Test for overall effect }\end{array}$ & $\begin{array}{r}0 \\
\text { plicable } \\
\text { Not appli }\end{array}$ & cable & 0 & & & & & & & \\
\hline \multicolumn{11}{|l|}{ 1.3.2 Sin anestesista } \\
\hline Kongkam, 2008 & 2 & 67 & 7 & 67 & $42.3 \%$ & $0.29[0.06,1.33]$ & & & - & \\
\hline RIPHAUS, 2009 & 3 & 43 & 3 & 22 & $24.0 \%$ & $0.51[0.11,2.33]$ & & & & \\
\hline Vargo, 2002 & 0 & 38 & 3 & 37 & $21.4 \%$ & $0.14[0.01,2.60]$ & $\leftarrow$ & & & \\
\hline $\begin{array}{l}\text { Wehrmann, } 1999 \\
\text { Subtotal }(95 \% \mathrm{Cl})\end{array}$ & 5 & $\begin{array}{l}100 \\
248\end{array}$ & 2 & $\begin{array}{r}98 \\
224\end{array}$ & $\begin{array}{r}12.2 \% \\
100.0 \%\end{array}$ & $\begin{array}{r}2.45[0.49,12.33] \\
0.57[0.27,1.24]\end{array}$ & & & & \\
\hline \multicolumn{11}{|c|}{$\begin{array}{l}\text { Heterogeneity. } \mathrm{Chi}^{2}=4.81, \mathrm{df}=3(P=0.19) ; \mathrm{I}^{2}=38 \% \\
\text { Test for overall effect: } Z=1.42(P=0.16)\end{array}$} \\
\hline Total $(95 \% \mathrm{Cl})$ & & 300 & & 276 & $100.0 \%$ & $0.57[0.27,1.24]$ & & & & \\
\hline Total events & 10 & & 15 & & & & & & & \\
\hline $\begin{array}{l}\text { Heterogeneity. } \mathrm{Chi}^{2}= \\
\text { Test for overall effect } \\
\text { Test for subqroup dif }\end{array}$ & $\begin{array}{l}4.81, \mathrm{df}= \\
\mathrm{Z}=1.42 \\
\text { erences: }\end{array}$ & $\begin{array}{l}3(P= \\
(P=0.1 \\
\text { Not app }\end{array}$ & $\begin{array}{l}0.19) ;\left.\right|^{2}=38 \% \\
\text { 16) } \\
\text { plicable }\end{array}$ & & & & 0.01 & $\begin{array}{cc}0.1 \\
\text { Favor Propofol }\end{array}$ & Favor ST & $\overrightarrow{100}$ \\
\hline
\end{tabular}

\section{Caption}

Forest plot of comparison: 1 Propofol v/s sedacion tradicional, outcome: 1.3 Bradicardia.

Figura 4. 


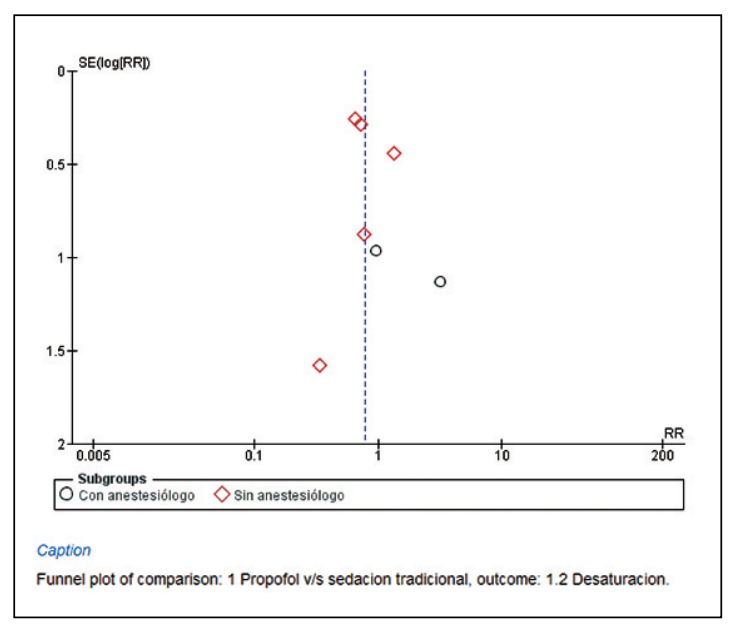

Figura 5.

\section{Discusión}

De acuerdo con lo descrito en la literatura, los resultados de nuestra revisión sistemática y posterior metaanálisis sugieren que el uso de propofol comparado con sedación tradicional para procedimientos endoscópicos altos no aumenta el riesgo de complicaciones cardiopulmonares.

Cabe señalar que a diferencia de nuestra pregunta de investigación original, evaluando el impacto de la sedación en procedimientos endoscópicos en general, la evidencia obtenida aplica exclusivamente a procedimientos endoscópicos altos, por carecer de estudios metodológicamente adecuados en endoscopias digestivas bajas. Sin embargo, Singh et al., en el año 2011 en una revisión Cochrane ${ }^{4}$, concluyeron que la sedación con propofol para colonoscopia no aumenta los efectos adversos comparado con sedación tradicional, no obstante en ese metaanálisis se incluyeron trabajos con propofol más coadyuvantes en la misma rama. Para estudiar los riesgos del propofol usado en forma exclusiva, nosotros decidimos incluir trabajos en donde se comparara propofol solo versus sedación tradicional.

Chiang et al. ${ }^{7}$, en el año 2013, compararon sedación con propofol en modalidad TCI (target controlled infusion) versus MCI (manual controlled infusion) para endoscopia digestiva; Los autores concluyen que usando TCI se disminuye en un 50\% el riesgo de desaturación. Interesantemente, en todos los trabajos incluidos en nuestra revisión el método de administración de propofol fue un bolo inicial de 30 a $50 \mathrm{mg}$ seguido por bolos de 10 a $20 \mathrm{mg}$ según necesidad, sin ajustes por peso de los pacientes. Considerando el estrecho margen terapéutico del propofol en términos de sedación y el riesgo de ap-
Tabla 3. Análisis de sensibilidad del efecto de cada estudio en el RR agrupado, al excluir un estudio a la vez y evaluar el efecto de los trabajos restantes, para hipoxia

\begin{tabular}{|lc|}
\hline Estudio excluido & $\begin{array}{c}\text { RR (IC 95\%) de los estudios } \\
\text { restantes }\end{array}$ \\
\hline Agrawal & $0,75[0,54-1,05]$ \\
\hline Khamaysi & $0,77[0,55-1,08]$ \\
\hline Kongkam & $0,81[0,54-1,21]$ \\
\hline Riphaus & $0,78[0,56-1,09]$ \\
\hline Vargo & $0,65[0,39-1,07]$ \\
\hline Wehrmann & $0,71[0,50-1,01]$ \\
\hline Weston & $0,79[0,56-1,09]$ \\
\hline Zuo & $0,78[0,56-1,08]$ \\
\hline
\end{tabular}

nea/hipopnea es un dato llamativo. Probablemente usando TCI, el riesgo de eventos asociados a una potencial sobredosificación de propofol hubiese sido menor. Sin embargo, por criterios de inclusión y calidad metodológica los trabajos con TCI quedaron excluidos de nuestro análisis.

Rex et al. ${ }^{3}$, analizaron retrospectivamente alrededor de 36.000 casos de sedaciones con propofol para procedimientos endoscópicos administradas por equipos de endoscopistas y enfermeras entrenadas, concluyendo que la administración de propofol por no anestesiólogos para sedación en endoscopia es segura. Sin embargo, este fue un estudio observacional, de carácter retrospectivo, y en la actualidad no hay ECR que comparen la seguridad de la administración de propofol por anestesiólogos versus no anestesiólogos. En nuestra revisión, al hacer un análisis de subgrupo según operador, no hubo diferencia significativa para ninguno de los efectos adversos medidos. Sin embargo, existe una tendencia a favor de que el uso de propofol se asocie a más incidencia hipoxia cuando el operador es un anestesiólogo, comparado con otro tipo de operadores. Esta observación puede resultar contraintuitiva, pero que puede explicarse porque los pacientes manejados con propofol por anestesiólogos tenían mayores niveles de sedación y mayor comorbilidad.

Nuestro trabajo presenta varias limitaciones. Una de ellas es la heterogeneidad en la medición de profundidad anestésica. En general se mide a través de escalas subjetivas aplicadas por el endoscopista o anestesiólogo, no siendo la misma en todos los trabajos incluidos. No en todos los trabajos se hizo un análisis estadístico de la diferencia en nivel de sedación entre el grupo propofol y sedación 
tradicional. Esto hace difícil comparar el nivel de sedación entre los artículos y sacar conclusiones al respecto.

\section{Conclusión}

El uso de propofol comparado con sedación tradicional para procedimientos endoscópicos altos no aumenta el riesgo de complicaciones cardiopulmonares. En los estudios analizados no hubo diferencias significativas al analizar el profesional a cargo de la sedación, pese a que existe una tendencia a favor de que el propofol genere más hipoxia cuando el operador es un anestesiólogo, comparado con otro tipo de operadores. Este efecto paradójico puede ser explicado por la sedación más profunda y comorbilidad de los pacientes en este subgrupo. Se necesitan más trabajos metodológicamente aptos que evalúen el impacto del operador según tipo de sedación en endoscopia.

\section{Referencias}

1. Fanti L, Testoni PA. Sedation and analgesia in gastrointestinal endoscopy: what's new? World J Gastroenterol. 2010;16:2451-7.

2. Gross JB, Bailey PL, Connis RT, Cote CJ, Davis FG, Epstein BS, et al. Practice Guidelines for Sedation and Analgesia by Non-Anesthesiologists. Anesthesiology 2002;96:1004-17.

3. Rex DK, Heuss LT, Walker JA, Qi R. Trained registered nurses/endoscopy teams can administer propofol safely for endoscopy. Gastroenterology 2005;129:1384-91.

4. Singh H, Poluha W, Cheung M, Choptain N, Baron KI, Taback SP. Propofol for sedation during colonoscopy. Cochrane Database Syst Rev 2008;4:Cd006268.

5. Jadad AR, Moore RA, Carroll D, Jenkinson C, Reynolds DJ, Gavaghan DJ, et al. Assessing the quality of reports of randomized clinical trials: is blinding necessary? Control Clin Trials 1996;17:1-12.

6. Schulz KF, Altman DG, Moher D. CONSORT 2010 statement: updated guidelines for reporting parallel group randomized trials. Ann Intern Med. 2010;152:726-32.
7. Chiang $\mathrm{MH}$, Wu SC, You CH, Wu KL, Chiu YC, Ma $\mathrm{CW}$, et al. Target-controlled infusion vs. manually controlled infusion of propofol with alfentanil for bidirectional endoscopy: a randomized controlled trial. Endoscopy 2013;45:907-14.

8. Agrawal A, Sharma BC, Sharma P, Uppal R, Sarin SK. Randomized controlled trial for endoscopy with propofol versus midazolam on psychometric tests and critical flicker frequency in people with cirrhosis. J Gastroenterol Hepatol. 2012;27:1726-32.

9. Khamaysi I, William N, Olga A, Alex I, Vladimir M, Kamal D, et al. Sub-clinical hepatic encephalopathy in cirrhotic patients is not aggravated by sedation with propofol compared to midazolam: a randomized controlled study. J Hepatol. 2011;54:72-7.

10. Kongkam P, Rerknimitr R, Punyathavorn S, SitthiAmorn C, Ponauthai Y, Prempracha N, et al. Propofol infusion versus intermittent meperidine and midazolam injection for conscious sedation in ERCP. J Gastrointestin Liver Dis. 2008;17:291-7.

11. Riphaus A, Lechowicz I, Frenz MB, Wehrmann T. Propofol sedation for upper gastrointestinal endoscopy in patients with liver cirrhosis as an alternative to midazolam to avoid acute deterioration of minimal encephalopathy: a randomized, controlled study. Scand J Gastroenterol. 2009;44:1244-51.

12. Vargo JJ, Zuccaro G, Jr., Dumot JA, Shermock KM, Morrow JB, Conwell DL, et al. Gastroenterologistadministered propofol versus meperidine and midazolam for advanced upper endoscopy: a prospective, randomized trial. Gastroenterology 2002;123:8-16.

13. Wehrmann T, Kokabpick S, Lembcke B, Caspary WF, Seifert H. Efficacy and safety of intravenous propofol sedation during routine ERCP: a prospective, controlled study. Gastrointest Endosc. 1999;49:677-83.

14. Weston BR, Chadalawada V, Chalasani N, Kwo P, Overley CA, Symms M, et al. Nurse-administered propofol versus midazolam and meperidine for upper endoscopy in cirrhotic patients. Am J Gastroenterol. 2003;98:24407.

15. Zuo XL, Li Z, Liu XP, Li CQ, Ji R, Wang P, et al. Propofol vs midazolam plus fentanyl for upper gastrointestinal endomicroscopy: a randomized trial. World J Gastroenterol. 2012;18:1814-21. 